Journal of mathematics and computer Science $\quad 7$ (2013) 80 - 88

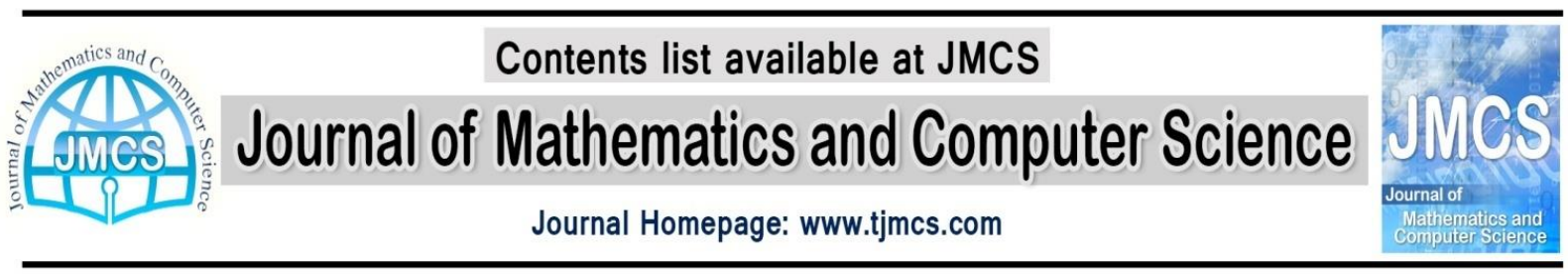

\title{
On fuzzy isomorphisms theorems of $\Gamma$-Hypernear-rings
}

\author{
E. Hendukolaie ${ }^{1}$, M. Aliakbarnia. Omran ${ }^{2}$, Y. Nasabi ${ }^{3}$ \\ ${ }^{1}$ Young Researchers Club, Islamic Azad University, Ayatollah Amoli Branch, Amol, Iran, \\ Edrishendoii@gmail.com \\ ${ }^{2}$ Amol Institution of higher education, Amol, Iran, \\ Mehdiomran@gmail.com \\ ${ }^{3}$ Young Researchers Club, Islamic Azad University, Ayatollah Amoli Branch, Amol, Iran, \\ Yaser.nasabi@yahoo.com
}

Article history:

Received February 2013

Accepted March 2013

Available online April 2013

Abstract
In this paper, first derived three isomorphism theorems of $\Gamma$-hypernear-rings. Also, by fuzzy $\Gamma$-hyperideals, We consider three fuzzy isomorphism theorems of $\Gamma$-hypernear-rings.

Keywords: $\Gamma$-Hypernear-rings, (fuzzy) isomorphism theorem, $\Gamma$-hyperideals, fuzzy $\Gamma$ -hyperideals.

\section{Introduction}

The theory of algebraic hyperstructures (or hypersystems) is a well-established branch of classical algebraic theory. In the literature, the theory of hyperstructure was first initiated by Marty in 1934 [20] when he defined the hypergroups and began to investigate their properties with applications to groups, rational fractions and algebraic functions. Later on, many people have observed that the theory of hyperstructures also have many applications in both pure and applied sciences, for example, semi-hypergroups are the simplest algebraic hyperstructures which possess the properties of closure and associativity. Some review of the theory of hyperstructures can be found in [4, 22], respectively. In a recent monograph of Corsini and Leoreanu [4], the authors have collected numerous applications of algebraic hyperstructures, especially those from the following subjects for the past fifteen years: geometry, hypergraphs, binary relations, lattices, fuzzy sets and rough sets, automata, cryptography, codes, median algebras, relation algebras, artificial intelligence and probabilities. The fuzzy sets and hyperstructures introduced by Zadeh [24] and Marty, respectively, are now extensively applied to many disciplines. The relationships between the fuzzy sets and algebraic hyperstructures have been considered by Ameri, Cristea, Corsini, Davvaz, Leoreanu, Zhan and many other researchers. The reader is refereed to $[5,9,10]$. The concept of $\Gamma$-rings is introduced by Barnes [3]. After that, this concept was discussed further by some researchers. The notion of 
fuzzy ideals in a $\Gamma$-ring was introduced by Jun and Lee in [23]. They studied some preliminary properties of fuzzy ideals of $\Gamma$-rings. Jun [15] defined fuzzy prime ideals of a $\Gamma$-ring and obtained a number of characterizations for a fuzzy ideal to be a fuzzy prime ideal. In particular, Dutta and Chanda [12] studied the structures of the set of fuzzy ideals of a $\Gamma$-ring. Ma et al. [19] considered the characterization of fuzzy h-ideals in h-hemiregular and h-semisimple $\Gamma$ -hemirings. Recently, Ameri et al. [2] considered the concept of fuzzy hyperideals of $\Gamma$ -hyperrings. By a different way of [2], Yin et al. [23] investigated some new results on $\Gamma$ -hyperrings. In particular, Davvaz et al. [11] considered the properties of $\Gamma$-hypernear-rings, and derived some related results.

In this paper, We will discuss the properties of $\Gamma$-hypernear-rings. In Section 2, we recall some basic concepts of $\Gamma$-hypernear-rings. In Section 3, we derive three isomorphism theorems of $\Gamma$-hypernear-rings. In particular, We establish three fuzzy isomorphism theorems of $\Gamma$ -hypernear-rings in Section 4.

\section{Preliminaries}

Firstly,let me introduce some preliminary concepts:

Definition 2.1 A hypergroupoid is a pair $(H,+)$ consisting of a nonempty set $H$ together with a hyperoperation $+: H \times H \rightarrow 2^{H} \backslash \varnothing$.

If $A$ and $B$ are nonempty subsets of $H$, then we denote:

$$
A+B=\bigcup_{a \in A, b \in B} a+b, \quad A+x=A+\{x\}, \quad x+B=\{x\}+B .
$$

A hypergroupoid $(H,+)$ is called a semihypergroup if for all $x, y, z$ of $H$ we have $(x+y)+z=x+(y+z)$, which means that

$$
\bigcup_{u \in x+y} u+z=\bigcup_{v \in y+z} x+v
$$

An element $e$ of $H$ is called an identity (scalar identity) of $(H,+)$ if for all $a \in H$, we have $a \in(e+a) \cap(e+a), \quad(\{a\}=(e+a) \cap(e+a))$. A hypergroup is a semihypergroup such that for all $x \in H$, we have $x+H=H=H+x$.

A subhypergroup $(K,+)$ of $(H,+)$ is a nonempty set $K$, such that for all $k \in K$, we have $k+K=K=k+K$.

Definition 2.2 [8] A hypergroupoid $(R,+)$ is a quasicanonical hypergroup if the following conditions hold:

(i) $(x+y)+z=x+(y+z), \quad \forall x, y, z \in R$;

(ii) $\exists 0 \in R$ such that $x+0=x=0+x, \quad \forall x \in R$;

(iii) $\forall x \in R, \quad \exists ! x^{\prime} \in R$ such that $0 \in\left(x+x^{\prime}\right) \cap\left(x^{\prime}+x\right)$;

(iv) $\forall x, y, z \in R$ and $z \in x+y \Rightarrow x \in z+(-y), \quad y \in(-x)+z$.

the following equalities follow easily from the axioms:

$$
-(-x)=x \text { and }-(x+y)=-x-y \text {. }
$$

Note that in the rest of the paper we will frequently write singletons without braces.

Definition 2.3 [8] The triple $(R,+, \cdot)$ is a hypernear-ring if: 
(1) $(R,+)$ is a quasicanonical hypergroup, i.e. the following axioms hold for $(R,+)$ :

(i) $(x+y)+z=x+(y+z), \quad \forall x, y, z \in R$;

(ii) $\exists 0 \in R$ such that $x+0=x=0+x, \quad \forall x \in R$;

(iii) $\forall x \in R, \quad \exists ! x^{\prime} \in R$ such that $0 \in\left(x+x^{\prime}\right) \cap\left(x^{\prime}+x\right)$;

(iv) $\forall x, y, z \in R$ and $z \in x+y \Rightarrow x \in z+(-y), \quad y \in(-x)+z$.

(2) $(R, \cdot)$ is a semihypergroup having 0 as a right absorbing element, i.e. $0 \cdot x=0$, $\forall x \in R$;

(3) $(x+y) \cdot z=x \cdot z+y \cdot z, \quad \forall x, y, z \in R$.

A subhypergroup $A \subseteq H$ is normal if we have $x+A-x \subseteq A$.

Precisely speaking,it is a left hypernear-ring because it satisfies the left distributive law. I will use the word hypernear-ring to mean "left hypernear-ring".

We note that if $x \in R$ and $\mathrm{A}$; $\mathrm{B}$ are non-empty subsets of $\mathrm{R}$, then by $A+B ; A+x$ and $x+B$ we can deduce that $A+B=\bigcup_{a \in A, b \in B} a+b, \quad A+x=A+\{x\}, \quad x+B=\{x\}+B$, respectively. Also, for all $x ; y \in R$, we have $-(-x)=x ; 0=-0$, where 0 is unique and $-(x+y)=-y-x$.

A subhypergroup $A \subseteq R$ is called normal if for all $x \in R$, we have $x+A-x \subseteq A$.

Definition 2.4 [8]A normal subhypergroup $A$ of hypergroup $(R,+)$ is

(i) a left hyperideal of $R$ if $x \cdot A \in A$, for all $x \in R, a \in A$;

(ii) a right hyperideal of $R$ if $(x+A) \cdot y-x y \subseteq A$, for all $x, y \in R$;

(iii) a hyperideal of $R$ if $(x+A) \cdot y-x y \cup z \cdot A \subseteq A$, for all $x, y, z \in R$.

The concept of a fuzzy subhypernear-ring was first introduced by Davvaz in [14] which is a generalized concept of a fuzzy subnear-ring (ideal) in a near-ring. I now formulate the following definitions:

Definition 2.5 [8] Let $(R,+, \quad \cdot)$ be a hypernear-ring. Then we call a fuzzy set $\mu$ of $R$ a fuzzy subhypernear-ring of $R$ if it satisfies the following inequalities:

(1a) $\min \{\mu(x), \mu(y)\} \leq \inf _{z \in x+y} \mu(z)$; for all $x ; y \in R$,

(1b) $\mu(x) \leq \mu(-x)$, for all $x \in R$,

(2) $\min \{\mu(x), \mu(y)\} \leq \mu(x y)$, for all $x ; y \in R$.

Furthermore,we call $\mu$ a fuzzy hyperideal of $\mathrm{R}$ if $\mu$ is a fuzzy subhypernear-ring of $\mathrm{R}$ and we have;

(3) $\mu(y) \leq i n_{f_{x \in+y-x}} \mu(z)$; for all $x ; y \in R$,

(4) $\mu(y) \leq \mu(x y)$,for all $x ; y \in R$.

(5) $\mu(i) \leq i n f_{(x \in+i) y-x y} \mu(z)$; for all $x ; y ; i \in R$.

Let $\mu$ be a fuzzy set. For every $t \in[0,1]$, the set $\mu_{t}=\{x \in R \mid \mu(x) \geq t\}$ is called the level subset of $\mu$.

Definition 2.6 [11] A $\Gamma$-hypernear-ring is a triple $(M,+, \Gamma)$, where

(i) $\Gamma$ is a non-empty set of binary operations such that $(M,+, \alpha)$, is ahypernear-ring for each $\alpha \in \Gamma$,

(ii) $\quad x \alpha(y \beta z)=(x \alpha y) \beta z$ for all $x ; y ; z \in M$ and $\alpha ; \beta \in \Gamma$.

In what follows, we use $\mathrm{M}$ to denote a $\Gamma$-hypernear-ring unless other wise specified. 
Example 2.7 Let $M=\{0, a, b\}$ and $\Gamma$ be the non-empty set of binary operations such that $\alpha, \beta \in \Gamma$ are defined as follows:

$\begin{array}{cccc}+ & 0 & a & b \\ 0 & \{0\} & \{a\} & \{b\} \\ a & \{a\} & \{0, a, b\} & \{a, b\} \\ b & \{b\} & \{a, b\} & \{0, a, b\}\end{array}$

Then $(M,+, \Gamma)$ is a $\Gamma$-hypernear-ring.

Example 2.8 Let $R$ be a near-ring and $M$ be a right $R$-module. We define the hyperoperation + on $M$ by $x+y=\{x+y\}$,for each $x ; y \in M$. Then $(M,+)$ is a hypergroup.If is a non-empty subset of $\operatorname{Hom}_{R}(M, R)$, then $(M,+, \Gamma)$ is a hypernear-ring for each $\alpha \in \Gamma$ and $x \alpha(y \beta z)=x(\alpha(y \beta(z))=x(\alpha(y) \beta(z))=(x \alpha(y)) \beta(z)=(x \alpha y) \beta z$ for every $\alpha, \beta \in \Gamma$. Hence $(M,+, \Gamma)$ is a $\Gamma$-hypernear-ring.

Definition 2.9 Let $(R,+, \Gamma)$ be a $\Gamma$-hypernear-ring. Then we call a fuzzy set $\mu$ of $R$ a fuzzy $\Gamma$-subhypernear-ring of $R$ if it satisfies the following inequalities:

(1a) $\min \{\mu(x), \mu(y)\} \leq \inf _{z \in x+y} \mu(z)$; for all $x ; y \in R$,

(1b) $\mu(x) \leq \mu(-x)$, for all $x \in R$,

(2) $\max \{\mu(x), \mu(y)\} \leq \mu(x \alpha y)$,for all $x ; y \in R$ and $\alpha \in \Gamma$.

Furthermore,we call $\mu$ a fuzzy $\Gamma$-hyperideal of $\mathrm{R}$ if $\mu$ is a fuzzy $\Gamma$-subhypernear-ring of $\mathrm{R}$ and we have;

$$
\begin{aligned}
& \mu(y) \leq i n_{z \in x+y-x} \mu(z) ; \text { for all } x ; y \in R, \\
& \mu(y) \leq \mu(x \alpha y), \text { for all } x ; y \in R \text { and } \alpha \in \Gamma . \\
& \mu(i) \leq i n_{z \in(x+i) y-x y} \mu(z) ; \text { for all } x ; y ; i \in R .
\end{aligned}
$$

\section{3 isomorphism theorem}

In this section, Some definitions and isomorphism theorems of $\Gamma$-hypernear-rings are stated that will be used in next section. Note that isomorphism theorems are proved in []. Now,we recall the following concept:

Definition 3.1 [11] If $M$ and $M^{\prime}$ be two $\Gamma$-hypernear-rings, then a mapping $f: M \rightarrow M^{\prime}$ is called a $\Gamma$-hypernear-ring homomorphism if for every $\alpha, \beta \in \Gamma$, we have:

$$
f(x+y)=f(x)+f(y), f(x \alpha y)=f(x) \alpha f(y) .
$$

Clearly,a $\Gamma$-hypernear-ring homomorphism $f$ is an isomorphism if $f$ is injective and surjective. We write $M \cong M^{\prime}$ if $M$ isomorphic to $M^{\prime}$.

Definition 3.2 I $I$ is a hyperideal of a hypernear-ring $R$, then we define the relation $x \equiv y(\bmod I)$ $\Leftrightarrow(x-y) \cap I \neq \varnothing$.

The relation is denoted by $x I^{*} y$. This is a congruence relation on $\mathrm{M}$.

The class $x+I$ is represented by $x$ and we denote it with $I^{*}(x)$. Moreover, $I^{*}(x)=I^{*}(y)$ 
if and only if $x \equiv y(\bmod I)$. We can define $M / I$ as follows:

$$
M / I=\left\{I^{*}(x) \mid x \in M\right\} .
$$

Define a hyperoperation $\oplus$ and an operation $\mathrm{e}$ on $M / I$ by

$$
\begin{aligned}
& I^{*}(x) \oplus I^{*}(y)=\left\{I^{*}(z) \mid z \in I^{*}(x)+I^{*}(y)\right\}, \\
& I^{*}(x) \mathrm{e}_{\alpha} I^{*}(y)=I^{*}(x \alpha y), \text { for all } I^{*}(x), I^{*}(y) \in M / I .
\end{aligned}
$$

From the above discussion, we can get the following theorem:

Theorem $3.3(M / I, \oplus, e)$ is a $\Gamma$-hypernear-ring.

Now, we establish three Isomorphism Theorems of $\Gamma$-hypernear-ring.

Theorem 3.4 [11] (First Isomorphism Theorem). Let $f$ be a $\Gamma$-hypernear-ring homomorphism from $M_{1}$ into $M_{2}$ with kernel $K$ such that $K$ is a hyperideal of $M_{1}$. Then we have

$$
M_{1} / K^{*} \cong \operatorname{Imf} \text {. }
$$

Theorem 3.5 [11] (Second Isomorphism Theorem). If $A$ and $B$ be two hyperideals of $M$, then we have

$$
A /(A \cap B)^{*} \cong(A+B) / B^{*}
$$

Theorem 3.6 [11] (Third Isomorphism Theorem). If $A$ and $B$ be two hyperideals of $M$ such that $A \subseteq B$, then $B / A^{*}$ is a hyperideal of $M / A^{*}$ and

$$
\left(M / A^{*}\right) /\left(B / A^{*}\right) \cong\left(M / B^{*}\right) .
$$

\section{The fuzzy isomorphisms theorem}

In this section, we establish fuzzy isomorphism theorems of $\Gamma$-hypernear-rings.

Definition 4.1 Let $\mu$ be a fuzzy $\Gamma$-hyperideal of $R$. Define the following relation:

$$
x \equiv y(\bmod \mu) \text { if and only if there exists } r \in x-y \text { such that } \mu(r)=\mu(0) \text {. }
$$

We usually denote the above relation by $\mu^{*}$. For this relation, we have the following lemmas. The proofs are obvious.

Lemma 4.2 The relation $\mu^{*}$ is an equivalence relation

Lemma 4.3 If $x \mu^{*} y$, then $\mu(x)=\mu(y)$.

Let $\mu^{*}[x]$ be the equivalence class of $x$. We denote by $R / \mu$ the set of all equivalence classes, i.e., $R / \mu=\left\{\mu^{*}[x] \mid x \in R\right\}$. Define the following two operations in $R / \mu$ :

$$
\begin{aligned}
& \mu^{*}[x]\left(\mu^{*}[y]=\left\{\mu^{*}[z] \mid z \in \mu^{*}[x] \oplus \mu^{*}[y]\right\} ;\right. \\
& \mu^{*}[x] \mathrm{e}_{\alpha} \mu^{*}[y]=\mu^{*}[x \alpha y] .
\end{aligned}
$$

Since $\mu^{*}$ is regular, then we can deduce the following result:

Theorem $4.4\left(R / \mu,\left(, \mathrm{e}_{\alpha}\right)\right.$ is a $\Gamma$-hypernear-ring.

Proof. Let $f: R \rightarrow R^{\prime}$ be a map and $\mu, v$ be the fuzzy sets of $R, R^{\prime}$, respectively. Then the image $f(\mu)$ of $\mu$ is the fuzzy subset of $R^{\prime}$ define by 


$$
f(\mu)(y)= \begin{cases}\operatorname{Sup}_{x \in f^{-1}(y)}\{\mu(x)\} & \text { iff } f^{-1}(y) \neq \varnothing \\ 0 & \text { otherwise }\end{cases}
$$

for all $y \in R^{\prime}$. The inverse image $f^{-1}(v)$ of $v$ is the fuzzy set of $\mathrm{R}$ defined by

$$
f^{-1}(v)(x)=v(f(x)), \text { for all } x \in R
$$

The following two lemmas are easily obtained and we omit the details.

Lemma 4.5 Let $f: R \rightarrow R$ be a $\Gamma$-hypernear-ring homomorphism. If $\mu$ is a fuzzy $\Gamma$ -hyperideal of $R$, then $f(\mu)$ is a fuzzy $\Gamma$-hyperideal of $R^{\prime}$.

Lemma 4.6 Let $f: R \rightarrow R^{\prime}$ be a $\Gamma$-hypernear-ring homomorphism. If $\mu$ and $v$ be two fuzzy $\Gamma$-hyperideal of $R$ and $R^{\prime}$, respectively.

(i) If $\mathrm{f}$ is an epimorphism, then $f\left(f^{-1}(v)\right)=v$;

(ii) If $\mu$ is a constant on $\operatorname{Kerf}$, then $f^{-1}(f(\mu))=\mu$.

Let $\mu$ be a fuzzy $\Gamma$-hyperideal of $\mathrm{R}$. We denote $R_{\mu}=\{x \in R \mid \mu(x)=\mu(0)\}$. Clearly, $R_{\mu}$ is a $\Gamma$-hyperideal of $R$.

Theorem 4.7 (First fuzzy isomorphism theorem). Let $f: R \rightarrow R^{\prime}$ be an epimorphism of $\Gamma$ -hypernear-rings and $\mu$ be a fuzzy $\Gamma$-hyperideal of $R$ with $R_{\mu} \supseteq \operatorname{Kerf}$. Then we have $R / \mu \cong R^{\prime} / f(\mu)$.

Proof. First, we know that $R / \mu$ and $R^{\prime} / f(\mu)$ are $\Gamma$-hypernear-rings. We define

$$
\lambda: R / \mu \rightarrow R^{\prime} / f(\mu) \text { by } \lambda\left(\mu^{*}[x]\right)=f(\mu)^{*}[f(x)], \text { for all } x \in R .
$$

Notice that $\lambda$ is well defined. In fact, if $\mu^{*}[x]=\mu^{*}[y]$, then $\mu(x)=\mu(y)$ by Lemma 4.3. Since $R_{\mu} \supseteq \operatorname{Kerf}$, it follows that $\mu$ is a constant on Kerf. By Lemma 4.6(ii) $f^{-1}(f(\mu))=\mu \quad$.Thus we have $\quad\left(f^{-1}(f(\mu))(x)=\left(f^{-1}(f(\mu))(y) \quad\right.\right.$, i.e., $(f(\mu))(f(x))=(f(\mu))(f(y))$. Hence we have $f(\mu)^{*}[f(x)]=f(\mu)^{*}[f(y)]$. Moreover, we have

(i)

$$
\begin{aligned}
& \lambda\left(\mu^{*}[x]\left(\mu^{*}[y]\right)=\lambda\left(\left\{\mu^{*}[z] \mid z \in \mu^{*}[x] \oplus \mu^{*}[y]\right\}\right)\right. \\
& =\left\{f(\mu)^{*}[f(z)] \mid z \in \mu^{*}[x] \oplus \mu^{*}[y]\right\} \\
& =f(\mu)^{*}\left(f\left(\mu^{*}[x]\right)\right) \oplus f(\mu)^{*}\left(f\left(\mu^{*}[y]\right)\right) \\
& =\lambda\left(\mu^{*}[x] \oplus \mu^{*}[y]\right)
\end{aligned}
$$

(ii)

$$
\begin{aligned}
& \lambda\left(\mu^{*}[x] \mathrm{e}_{\alpha} \mu^{*}[y]\right)=\lambda\left(\mu^{*}[x \alpha y]\right) \\
& =f(\mu)^{*}(f(x \alpha y)) \\
& =f(\mu)^{*}(x \alpha y) \\
& =f(\mu)^{*}(f[x]) \alpha f(\mu)^{*}(f[y]) \\
& =\lambda\left(\mu^{*}[x]\right) \alpha \lambda\left(\mu^{*}[y]\right) .
\end{aligned}
$$

Hence, $\lambda$ is a $\Gamma$-hypernear-ring homomorphism. Clearly, $\lambda$ is an epimorphism. To show 
that $\lambda$ is a monomorphism, let $f(\mu)^{*}[f(x)]=f(\mu)^{*}[f(y)]$, then $(f(\mu))(f(x))=(f(\mu))(f(y))$, that is, $\left(f^{-1}(f(\mu))(x)=\left(f^{-1}(f(\mu))(y)\right.\right.$. Hence $\mu(x)=\mu(y)$, and so $\mu^{*}[x]=\mu^{*}[y]$. Therefore $R / \mu \cong R^{\prime} / f(\mu)$.

The following lemma is obvious.

Lemma 4.8 Let $f: R \rightarrow R^{\prime}$ be an epimorphism of $\Gamma$-hypernear-rings and $v$ be a fuzzy $\Gamma$ hyperideal of $R^{\prime}$, then $f^{-1}(v)$ is a fuzzy $\Gamma$-hyperideal of $R$.

Corollary 4.9 Let $f: R \rightarrow R^{\prime}$ be an epimorphism of $\Gamma$-hypernear-rings and $v$ be a fuzzy $\Gamma$ hyperideal of $R^{\prime}$, then we have $R / f^{-1}(v) \cong R^{\prime} / v$.

Proof. By Lemma 4.8, $f^{-1}(v)$ is a fuzzy $\Gamma$-hyperideal of $\mathrm{R}$ and so $R / f^{-1}(v)$ and $R^{\prime} / v$ are $\Gamma$-hypernear-rings. We check that $R_{f^{-1}(v)} \supseteq \operatorname{Kerf}$. In fact, for any $x \in \operatorname{Kerf}$, we have $f(x)=f(0)$, and so $v(f(x))=v(f(0))$, i.e., $\left(f^{-1}(v)(x)\right)=\left(f^{-1}(v)(0)\right)$. Hence $x \in R_{f^{-1}}(v)$, and so $R_{f^{-1}(v)} \supseteq \operatorname{Kerf}$. By Theorem 4.7, we have $R / f^{-1}(v) \cong R^{\prime} / v$. This completes the proof.

Now, we give the second fuzzy and third fuzzy isomorphism theorems. The following two lemmas are obvious and we omit the details.

Lemma 4.10 Let $A$ be a $\Gamma$-hyperideal of $R$ and $\mu$ be a fuzzy $\Gamma$-hyperideal of $R$. then

(i) If $\mu$ is restricted to $A$, then $\mu$ is a fuzzy $\Gamma$-hyperideal of $A$;

(ii) $A / \mu$ is a $\Gamma$-hyperideal of $R / \mu$.

Lemma 4.11 If $\mu$ and $v$ are any two fuzzy $\Gamma$-hyperideals of $R$, then so is $\mu \cap v$.

Theorem 4.12 (Second fuzzy isomorphism theorem). If $\mu$ and $v$ are any two fuzzy $\Gamma$ -hyperideals of $R$ with $\mu(0)=v(0)$, then $R_{\mu} /(\mu \cap v) \cong\left(R_{\mu}+R_{v}\right) / v$.

Proof. By Lemmas 4.10 and 4.11, $v$ and $\mu \cap v$ are two fuzzy $\Gamma$-hyperideals of $R_{\mu}+R_{v}$ and $R_{\mu}$, respectively. Thus $\left(R_{\mu}+R_{v}\right) / v$ and $R_{\mu} /(\mu \cap v)$ are both $\Gamma$-hyperideals.

Define $\delta: R_{\mu} \rightarrow\left(R_{\mu}+R_{v}\right) / v$ by $\delta(x)=v^{*}[x]$, for all $x \in R_{\mu}$. It is easy to see that $\delta$ is an epimorphism. We check that $\operatorname{Ker} \delta=\mu \cap \nu$.

$$
\begin{aligned}
& \operatorname{Ker} \delta=\left\{x \in R_{\mu} \mid \delta(x)=v^{*}[0]\right\} \\
& =\left\{x \in R_{\mu} \mid v^{*}[x]=v^{*}[0]\right\} \\
& =\left\{x \in R_{\mu} \mid v(x)=v(0)\right\} \\
& =\left\{x \in R_{\mu} \mid \mu(x)=\mu(0) v(0)=v(x)\right\} \\
& =\left\{x \in R_{\mu} \mid x \in R_{v}\right\} \\
& =\mu \cap v
\end{aligned}
$$

Therefore $R_{\mu} /(\mu \cap v) \cong\left(R_{\mu}+R_{v}\right) / v$. 
Theorem 4.13 (Third fuzzy isomorphism theorem). Let $\mu$ and $v$ be two fuzzy $\Gamma$-hyperideals of $R$ with $v \leq \mu$ and $\mu(0)=v(0)$, then $(R / v) /\left(R_{\mu} / v\right) \cong R / \mu$.

Proof. By Lemma 4.10(ii), we know that $R_{\mu} / v$ is a $\Gamma$-hyperideal of $R / v$. Define $\tau: R / v \rightarrow R / \mu$ by $\tau\left(v^{*}[x]\right)=\mu^{*}[x]$, for all $x \in R$. If $v^{*}[x]=v^{*}[y]$, for all $x, y \in R$, then there exists $r \in x-y$, such that $v(r)=v(0)$. Since $v \leq \mu$ and $\mu(0)=v(0)$, we have $\mu(r) \geq v(r)=v(0)=\mu(0)$, which implies that $\mu(r)=\mu(0)$, and so $\mu^{*}(x)=\mu^{*}(y)$. Hence, $\mathrm{f}$ is well defined. Moreover, we have

(i)

$$
\begin{aligned}
& \tau\left(v^{*}[x]\left(v^{*}[y]\right)=\tau\left(\left\{v^{*}[z] \mid z \in v^{*}[x] \oplus v^{*}[y]\right\}\right)\right. \\
& \left.=\left\{\mu^{*}[z)\right] \mid z \in v^{*}[x] \oplus v^{*}[y]\right\} \\
& =\mu^{*}\left[v^{*}[x]\right]\left(\mu^{*}\left[v^{*}[y]\right]\right. \\
& =\mu^{*}[x]\left(\mu^{*}[y]\right. \\
& =\tau\left(v^{*}[x]\right)\left(\tau\left(v^{*}[y]\right)\right.
\end{aligned}
$$

(ii)

$$
\begin{aligned}
& \tau\left(v^{*}[x] \mathrm{e}_{\alpha} v^{*}[y]\right)=\tau\left(v^{*}[x \alpha y]\right) \\
& =\mu^{*}[x \alpha y] \\
& =\mu^{*}[x] \mathrm{e}_{\alpha} \mu^{*}[y] \\
& =\tau\left(v^{*}[x]\right) \alpha \tau\left(v^{*}[y]\right)
\end{aligned}
$$

Hence, $f$ is a $\Gamma$-hypernear-ring homomorphism. Clearly, $f$ is an epimorphism. Next, we show that $\operatorname{Kerf}=R_{\mu} / v$. In fact,

$$
\begin{aligned}
& \operatorname{Ker} \tau=\left\{v^{*}[x] \in R / v \mid \tau\left(v^{*}[x]\right)=\mu^{*}[0]\right\} \\
& =\left\{v^{*}[x] \in R / v \mid \mu^{*}[x]=\mu^{*}[0]\right\} \\
& =\left\{v^{*}[x] \in R / v \mid \mu(x)=\mu(0)\right\} \\
& =\left\{v^{*}[x] \in R / v \mid x \in R_{\mu}\right\} \\
& =R_{\mu} / v .
\end{aligned}
$$

Therefore, from Theorem 4.7 it follows that $(R / v) /\left(R_{\mu} / v\right) \cong R / \mu$.

\section{References}

[1] R. Ameri, E. Hendoukolaii, Fuzzy Hypernear-rings, Ital. J. Pure Appl. Math. to appear.

[2] R. Ameri, H. Hedayati, A. Molaee, On fuzzy hyperideals of $\Gamma$-hyperrings, Iran J. Fuzzy Systems 6, 47-60 (2009).

[3] W.E. Barbes, On the $\Gamma$-rings of Nobusawa, Pacific J. Math. 18, 411-422 (1966).

[4] P. Corsini, V. Leoreanu, Applications of Hyperstructure Theory, Advances in Mathematics (Dordrecht), Kluwer Academic Publishers, Dordrecht, (2003).

[5] I. Cristea, B. Davvaz, Atanassov's intuitionistic fuzzy grade of hypergroups, Inform. Sci. 180, 
1506-1517 (2010).

[6] I. Cristea, S. Hoskova, Fuzzy pseudotopological hypergroupoids, Iran. J. Fuzzy Syst 6, 11-19 (2009).

[7] V. Dasic, Hypernear-rings, in: Proc. Fourth Int. Congress on AHA (1990), World Scientific, pp. 75-85 (1991).

[8] B. Davvaz, On hypernear-rings and fuzzy hyperideals, J.Fuzzy Math.7, 745 753, (1999).

[9] B. Davvaz, Isomorphism theorems on hyperrings, Indian J. Pure Appl. Math. 35 (3), 321-331 (2004).

[10] B. Davvaz, V. Leoreanu-Fotea, Hyperrings Theory and Applications, Int. Acad. Press, USA, (2007).

[11] B. Davvaz, J. Zhan, K.H. Kim, Fuzzy $\Gamma$-hypernear-rings, Comput. Math. Appl. 59, 2846-2853 (2010).

[12] T.K. Dutta, T. Chanda, Structures of fuzzy ideals of $\Gamma$-rings, Bull. Malays. Math. Sci. Soc. 28 (1), 9-15 (2005).

[13] E. Hendukolaii , On fuzzy homomorphisms between Hypernear-rings, tjmcs vol.2 no.4, 702-716 (2011).

[14] Y.B. Jun, On fuzzy prime ideals of $\Gamma$-rings, Soochow J. Math. 21 (1), 41-48 (1995).

[15] Y.B. Jun, C.Y. Lee, Fuzzy $\Gamma$-rings, Pusan Kyongnam Math. J. (now, East Asian Math. J.) 8 (2), 163-170 (1992).

[16] V. Leoreanu-Fotea, P. Corsini, Isomorphisms of hypergroups and of n-hypergroups with applications, Soft Comput. 13, 985-994 (2009).

[17] V. Leoreanu-Fotea, B. Davvaz, Fuzzy hyperrings, Fuzzy Sets Syst. 160, 2366-2378 (2009).

[18] X. Ma, J. Zhan, V. Leoreanu-Fotea, On (fuzzy) isomorphism theorems of $\Gamma$-hyperrings, Computers and Mathematics with Applications 60, 2594-2600 (2010).

[19] X. Ma, J. Zhan, Fuzzy h-ideals in h-hemiregular and h-semisimple $\Gamma$-hemirings, Neural Comput. Appl. 19, 477-485 (2010).

[20] F. Marty, Sur une generalization de la notion de groupe, in: 8th Congress Math. Scandianaves, Stockholm, pp. 45-49 (1934).

[21] M.K. Sen, R. Ameri, G. Chowdhury, Fuzzy hypersemigroups, Soft Comput. 12, 891-900 (2008).

[22] T. Vougiouklis, Hyperstructures and their Representations, Hadronic Press Inc., Palm Harbor, USA, (1994).

[23] Y. Yin, et al. A fuzzy view of $\Gamma$-hyperrings (submitted for publication).

[24] L.A. Zadeh, Fuzzy sets, Inform. Control 8, 338-353 (1965).

[25] J. Zhan, B. Davvaz, K.P. Shum, Generalized fuzzy hyperideals of hyperrings, Comput. Math. Appl. $56,1732-1740$ (2008).

[26] J. Zhan, B. Davvaz, K.P. Shum, Isomorphism theorems of hypermodules, Acta Math. Sinica (Chinese Ser.) 50, 909-914 (2007).

[27] J. Zhan, B. Davvaz, K.P. Shum, On fuzzy isomorphism theorems of hypermodules, Soft Comput. 11, 1053-1057 (2007).

[28] J. Zhan, B. Davvaz, K.P. Shum, A new view of fuzzy hypernear-rings, Inform. Sci. 178, 425-438 (2008). 